pathy. In his view, the central feature of the disorder was "an extraordinarily peculiar amnesia, in which the memory for recent events, those that have just happened, is principally involved, whereas the remote past is remem. bered fairly well". His observations were abundantly confirmed by others, though it soon became clear that this curious disorder is not exclusively linked with chronic alcoholism and may be regarded as a reaction of the brain to a wide variety of pathological agencies. It was therefore christened the Korsakoff syndrome, by which name it has since been generally known.

In view of the widespread preoccupation with the cerebral basis of memory, it is odd that the Korsakoff syndrome has not attracted greater scientific interest. For here we see a remarkable dissociation between memory, which is often most severely impaired, and other psychological functions, such as intelligence, which are little, if at all, affected. So clear-cut a dissociation cannot, so far as is known at present, be produced experimentally in animals, and may perhaps reflect a special characteristic of the human brain.

Dr. Talland presents an excellent account of the Korsakoff syndrome from the psychological point of view. Ho is particularly good on the historical aspect, and has made a real effort to understand the laborious German work on the subject, which at its best is extremely penetrating, though at its worst turgid and quasi-metaphysical. Indoed, Deranged Memory is the first really adequate digest of the literature on the Korsakoff syndrome so far to appear in English.

Included also are the results of a considerable number of controlled psychological experiments which the author has himself carried out with Korsakoff patients, some of which have already been reported elsewhere. These experiments have two aims: first, to ascertain the general psychological status of the Korsakoff patient, and secondly, to throw light on the nature of the memory defect itself. As regards the first aim, Dr. 'Talland shows, as have others before him, that the dofect of memory is seldom absolutely circumscribed. Minor abnormalities in perception, intelligence and reasoning can often be elicited by special tests, even if clinically inapparent. As regards the second aim, Dr. Talland is able to show that the memory loss cannot be exclusively ascribed to a failure of registration, of retention or of recall. All three phases of the memory process suffer, as does also associative learning. Indeed, all that one can really conclude from these experiments is that the rate of forgetting is enormously accelerated in Korsakoff patients, such that they become virtually unable to learn or to build up a coherent record of their present experience. As Sir Aubrey Lewis has well said, tho differences between the amnesic and the normal state are quantitative rather than quali. tative, and involve no factors not already known from the investigation of normal memory.

It is perhaps a pity that Dr. Talland pays so little attention to the neuropathology of the Korsakoff syn. drome and its relation to the more strictly circumscribed defects of memory that are now known to result from bilateral lesions in the hippocampal region of the brain. For, in these cases, one may see a relatively pure memory disorder, lacking the confabulation and disorientation so characteristic of the Korsakoff state. Indeed, the latter is unlikely to prove altogether simple, from the psychological point of view, and almost certainly involves defects in insight and judgment which are not necessarily linked with, lot alone derived from, the peculiar disorder of memory. These additional defects may well derive from subcortical brain damage, which has been established in many typical cases of alcoholic Korsakoff syndrome.

This interesting and unusual book may be commended to psychologists and others with an interest in memory and its disorders, It is well organized and clearly written, and will long remain a valuable source book. Unfortunately the price is rather high.

\section{RESPONSES OF INDIVIDUALS AND ENVIRONMENTS}

Psychobiological Approaches to Social Behavior

Edited by P. H. Leiderman and D. Shapiro. Pp. xv +203. (London : Tavistock Publications, Ltd., 1965.) $48 s$.

A SYMPOSIUM, sponsored jointly by the Department of Psychiatry of the Harvard Medical School and the U.S. Office of Naval Research, was held in 1963 to bring together individuals concerned with an increasingly significant field of psychological investigation, the impact of social and group environment on the emotional and bodily responses of man. It seems so apparent that psychological functioning must be influenced by the social environment that it is almost startling to note that the social psychological background is rarely specified in physiological investigation. Psychobiological Approaches to Social Behavior brings together data which would normally be spread through the literature of several disciplines. Among the interactions of the different life sciences which were described by various authors are the sensitivity of psychoendocrine systems to social and physical environment; plasma-lipid responses to leadership, conformity and deviation; muscular tension; physiological activation or psychological act?; the interaction of the cognitive and physiological determinants of emotional state; and the experimental exploration of the et-epimeletic or care-soliciting behavioural system.

Bringing together the proceedings of this symposium for publication in book form has been entrusted to Prof. P. H. Leiderman and Prof. D. Shapiro, well known for their work showing how social factors in small group interaction influence the galvanic skin potential. In a concluding chapter they discuss the implications for future research of the investigations reported and rightly suggest that there has already been established a methodological and conceptual framework for fruitful exchange between the psychological and the social sciences. The exchange will almost certainly includo a wide extension of suitable mathematical techniques. Future research should proceed from those studies which attempt to manipulate social variables by pre-experimental selection and arrangement of individuals. In the words of the editors, "small groups of two, three and four subjects could be studied under the varying effects of social approval and disapproval, success and failure, co-operation and competition, formal and informal social settings, conditions of isolation, and varying social relationships. Groups selected for personality type, patient category, occupational group, sex and ethnicity could be included in these different social environments". Other investigations could begin with the study of the socialization of physiological response levels during the course of maturation; useful information concerning individual and family structure could be obtained by physiological studies of family interaction during the course of development. Another area of research which would have an important bearing on psychosomatic medicine is the degree to which visceral responses can be learned or unlearned under different conditions of social reward. Equally, as the editors show, the relationship between pharmacological agents and social behaviour is almost unexplored. "A social situation is the only appropriate testing ground for assessing the value of a drug in influencing an individual's adaptation to his social environment." Other lines of research which are indicated from this fascinating integration of social and biological factors would stem from human evolution, genetics and ecology. The editors and authors could fairly claim that this report of their symposium is not only an important and exciting book but provides guidelines for much inter-disciplinary co-operation between workers in the psycho-biological fields of to-morrow.

T. H. Hawkins 